\title{
Smith predictor-based PI control of a wet granulation process
}

\author{
Tamir Shaqarin* \\ Mechanical Engineering Department, Tafila Technical University, Tafila 66110, Jordan
}

The need for prediction and reference updating in feedback control of a wet granulation process is addressed. The granulation process is often modeled as a multi-input multi-output (MIMO) linear model with dead-time. Industrial implementation of granulation process poses strict constraints on the process inputs \& outputs. The presence of dead-time and the physical necessity of the input-output constraints are the key challenges of the wet granulation control. These challenges motivated the use of model predictive control (MPC) for such processes. In this work, a Smith predictor-based proportional-integral (PI) controller is designed for the dead-time compensation. Accompanied with the reference updating method to handle the physical constraints. The regulation and reference tracking control problems are assessed via closed-loop simulations of the wet granulation model. The ability of the proposed control approach of dead-time compensation and coping with input/output constraints is rigorously proved. The current approach is compared to MPC of a similar granulation process and found superior in terms of output stability, performance and reference tracking.

Keywords: Granulation control, Dead-time compensation, Smith predictor, Reference updating, Constrained MIMO control

\section{Introduction}

In the last decade, the control of granulation is an active research field for the major benefits associated with pharmaceutical, agricultural, and chemical industries. Granulation is a process of making granules of fine particles with controlled properties such as attrition resistance, size uniformity, porosity, break-up rates, etc. Granulation process can be classified based on binder nature as dry or wet. In dry granulation, solid particles binders are added to the agitated powder then the adherence is stimulated by compaction and milling. On the other hand, wet granulation consisting of adding a liquid binder to the agitated powder blend, subsequently, the granules are processed and dried. The liquid binder is provided to the powder mixture by spraying, pouring, or dripping. Many reviews were reported on wet granulation in terms of granulator technologies, granule attributes, modeling and simulation, characterization tools, process control, and kinetics (Suresh et al., 2017; Iveson et al., 2001; Reynolds et al., 2005; Cameron et al., 2005; Burggraeve et al., 2013; Hansuld \& Briens, 2014). The attributes measures of granules are interconnected, mainly, to bulk density and particle size distribution (Pottmann et al., 2000). The oversized and undersized granules are crushed and recycled to the granulation process again. The oversized granules are those greater than an upper limit and undersized granules are those smaller than a lower limit. These limits are typically considered in order to attain a desired product quality (Pottmann et al., 2000). The mechanism of moisture and binder addition plays a significant role in average granule size. The spray nozzle locations and the moisture and binder ow rates through nozzles influence the particle growth rate and the granulation mechanism (Pottmann et al., 2000). Rankell et al. (1964) showed that the average granule size relates directly to the spray rate and inversely to the powder bed velocity. Tardos et al, (1997) reported that increasing the spray surface area will effectively narrow down the particle size distribution. Granulation improves the flow properties, reduces explosion risks, and gives well other specific performance aspects of the granules. The complexity and the difficulties encountered in inline/online measurement of product parameters make control of the processes a key difficulty because a small change to one variable affects several process responses. According to Merkus \& Meesters (2016), model based control overcomes this problem. Efficient process control plays an essential role in realizing the preferred attributes of granules. However, it requires complex feedback control approaches and in-line or on-line monitoring of the process. One important challenge in granulation process control is finding accurate mathematical models to simulate the entire process due to infinite dimensional character of the process (Buck et al., 2015). Thus, in general, a process control needs to be achieved through unified use of characterization methods. These methods track the changes in granule attributes with time and modifying process parameters accordingly (Suresh et al., 2017).

There are different control schemes for controlling granulation process. Pottmann et al., (2000) suggested using the model predictive control (MPC) for a drum granulation process. They fitted experimental data to linear discrete-time model for the process. Their control objectives are tracking the bulk density to a reference value and keeping the particle diameters within limits. Gatzke \& Doyle III (2001) controlled the same model of (Pottmann et al., 2000) using MPC with relaxed output constraints and prioritized control objectives. Sanders et al. (2009) presented a linear MPC valid for a nonlinear granulation process. The linearized state-space model was derived from a nonlinear discretized population balance model. They showed that MPC provides more stability than generic PID controller. Burggraeve et al. (2012) designed a feed-forward control strategy for a top-spray fluid bed granulation process. They also presented a partial least squares (PLS) model to predict the end product density. Closed-loop feedback control for continuous wet granulation in silico was tested by Singh et al. (2014).

Received on June 11, 2018; accepted on 20 August, 2018 Correspondence concerning this article should be addressed to Tamir Shaqarin (e-mail address: tshagareen@ttu.edu.jo).ORCiD ID of T. Shaqarin https://orcid.org/ 0000-0003-3327-3147 
They coupled controller parameter tuning strategy with an optimization strategy. The tuning strategy includes an integral of time absolute error method. The disturbance rejection capabilities were investigated. Model free control approaches such as fuzzy logic and artificial neural network were implemented to model and control granulation process (Petrovic et al., 2011). In present work, the control problem of a wet granulation process is addressed. A linear MIMO model with dead-time presented by Pottmann et al. (2000) is considered. A Smith (1957) predictor-based PI controller is used for dead-time compensation. In addition, the reference updating method is used to cope with the input/output constraints.

\section{Materials and Methods}

\subsection{Problem formulation}

Generally, enlargement of the particle size of the granules is mainly targeted by a granulation process among other physical properties of granular material. Figure 1 shows schematic of the wet granulation process studied herein. The spray nozzles flow rates of the liquid mixture $\left(u_{1}, u_{2}\right.$ and $\left.u_{3}\right)$ are the manipulated variables of the process. The liquid mixture that contains binding agent is introduced into particulate feed. The controlled variables of the process $\left(y_{1}, y_{2}\right.$ and $\left.y_{3}\right)$ are the bulk density of the product slurry, the product particle size of particles in the 5th percentile, and the product particle size of particles in the 90th percentile. Such process set-up was previously investigated and modelled by a linear MIMO system with dead-time. For example, a black-box MIMO model introduced by Pottmann et al. (2000) is considered here. This model was used as a benchmark by Gatzke \& Doyle III (2001) on which they implemented MPC to control the granulation. The model is represented by:

$$
\begin{aligned}
& G(s)=\left[\frac{k_{i, j}}{\tau_{i, j} s+1} e^{-\theta_{j} s}\right] \\
& G(s)=\left[\begin{array}{lll}
\frac{0.20}{2 s+1} e^{-3 s} & \frac{0.58}{2 s+1} e^{-3 s} & \frac{0.35}{2 s+1} e^{-3 s} \\
\frac{0.25}{3 s+1} e^{-3 s} & \frac{1.10}{3 s+1} e^{-3 s} & \frac{1.30}{3 s+1} e^{-3 s} \\
\frac{0.30}{4 s+1} e^{-3 s} & \frac{0.70}{4 s+1} e^{-3 s} & \frac{1.20}{4 s+1} e^{-3 s}
\end{array}\right]
\end{aligned}
$$

where $k$ is the steady state gain, $\tau$ time constant, $\theta$ dead-time, $i$ number of outputs and $j$ number of inputs. The steady state values for $\mathrm{y}_{1}, \mathrm{y}_{2}$ and $\mathrm{y}_{3}$ are 40,400, and 1620, and for $u_{1}, u_{2}$ and $u_{3}$ are 175,175 , and 245 , respectively. The model input and output values were normalized in non-dimensional units. More details on the aforementioned model can be found in (Pottmann et al. 2000; Gatzke \& Doyle III, 2001).

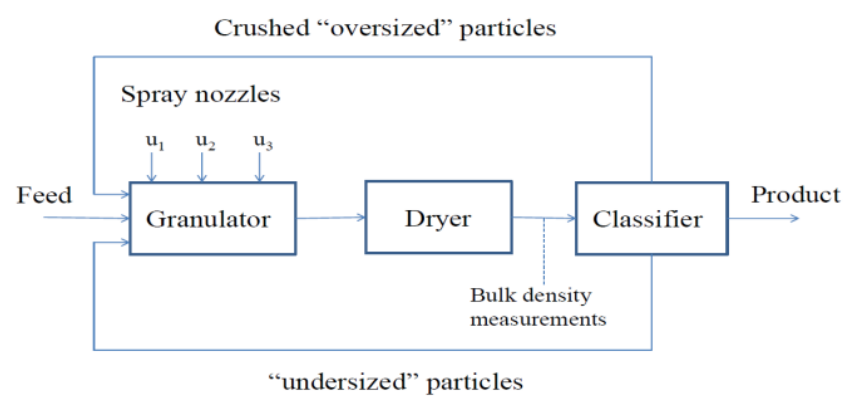

Fig. 1 Continuous wet granulation process (Pottmann et al., 2000)

\subsection{Controller design}

The control of granulation process requires the regulation of the bulk density to a certain set-point while keeping the product 9th percentile below 1650 and the product 5th percentile above 350. Meanwhile, all the nozzles flow rates must be maintained between 100 to 340 . The control of granulation process suffers from the dead-time problem and the input-output constraints. In the following Smith predictor-based PI controller is used for dead-time compensation. Furthermore, a reference updating technique is exploited to cope with the input/output constraints. Processes with large dead-time are key challenges in the classical control feedback loop as seen in Figure 2(a), this can be attributed to several reasons. Primarily, the control effort $(u)$ takes some time to have an effect in the controlled variable $(y)$. Moreover, the control effort that is generated based on real-time measurements attempts to correct past events. Furthermore, the effect of the disturbances is not 
detected by the controller until a significant time has passed. In general, large time-delay decreases the phase margins of classical controller that urges the closed-loop system toward instability (Normey-Rico, 2007).

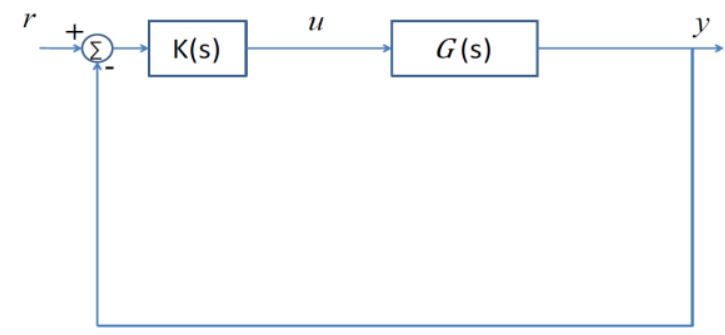

(a) Without Smith predictor

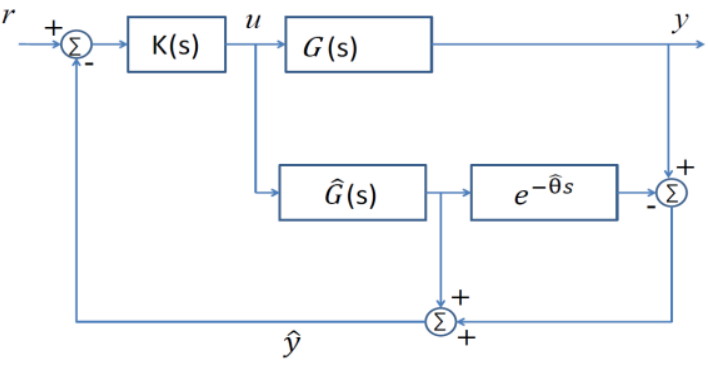

(b) With Smith predictor

Fig. 2 Closed-loop control structure.

The Smith (1957) predictor is the most common technique for dead-time compensation. The structure of this predictor is depicted in Figure 2. The controller output $u$ is fed through a model of the process and through the same model without dead-time. This way, the controller acts on a simulated process which behaves as if there was no dead-time in the process (in the ideal case of a perfect modeling). The MIMO granulation model in Eqn. (1) \& (2) can be substantially simplified as a MIMO system with single dead-time, such as

$$
G(s)=\left[\frac{k_{i, j}}{\tau_{i, j} s+1} e^{-\theta_{j} s}\right]:=\left[\frac{k_{i, j}}{\tau_{i, j} s+1}\right] e^{-\widehat{\theta} s}=\hat{G}(s) e^{-\widehat{\theta} s}
$$

Then, the model is decomposed into rational part $(\widehat{\mathrm{G}}(\mathrm{s}))$ and irrational part $\left(\mathrm{e}^{-\widehat{\theta} \mathrm{s}}\right)$. Such decomposition benefits from the fact that the time-delays $\theta_{j}$ in the aforementioned nominal model are all equal. From Figure $2(b)$, the predicted output $\hat{y}$ in the Laplace domain can be written as:

$$
\hat{Y}=G U-\hat{G} U e^{-\widehat{\theta} s}+\widehat{G} U
$$

In the nominal case $\left(G(s)=\hat{G}(s) e^{-\widehat{\theta} s}\right)$, then:

$$
\begin{aligned}
& \hat{Y}=\hat{G} U=\hat{G} K(R-\hat{Y}) \\
& \hat{Y}=(I+\hat{G} K)^{-1} \hat{G} K R
\end{aligned}
$$

That is used to compute the output $y$, such as:

$$
\begin{gathered}
Y=G U=G K(R-\hat{Y})=G K\left(R-(I+\hat{G} K)^{-1} \hat{G} K R\right) \\
Y=G K(I+\hat{G} K)^{-1} R
\end{gathered}
$$

where the closed-loop transfer function from $\mathrm{r}$ to $\mathrm{y}$ is $(I+\widehat{G} K)^{-1}$. This extension of Smith predictor to MIMO system with single dead-time ensures all the SP benefits for the SISO systems (Normey-Rico, 2007); the delay free characteristic equation $(\operatorname{det}(I+\widehat{G} K)=0)$, output prediction $(\widehat{Y}=\widehat{G} U)$ and ideal decoupled dynamic compensation $\left(G=\hat{G} e^{-\widehat{\theta} s}\right)$.

The controller $K$ is chosen as a diagonal matrix of PI controllers, such that:

$$
K(s)=\left[\begin{array}{ccc}
\frac{g_{1}\left(T_{1} s+1\right)}{T_{1} s} & 0 & 0 \\
0 & \frac{g_{2}\left(T_{2} s+1\right)}{T_{2} s} & 0 \\
0 & 0 & \frac{g_{3}\left(T_{3} s+1\right)}{T_{3} s}
\end{array}\right]
$$

The design of the PI controllers is based on the method presented in Normey-Rico (2007). The controller gain $g_{i}$ is selected to be proportional to the inverse of the diagonal model gain $k_{i i}$. Then it is tuned to have the desired performance characteristics. The 
integral time $T_{i}$ is selected to be equal to the diagonal model time constant $\tau_{i i}$. Then,

$$
g_{i} \propto \frac{1}{k_{i i}}, \quad T_{i}=\tau_{i i}
$$

The control of MIMO system is more difficult when imposing input/output constrains. Generally, controlling constrained MIMO systems motivates the use of MPC (Goodwin et al., 2005) or adaptive MPC (Landau et al., 2011). The control of the granulation process is a constrained MIMO control problem. Fortunately, only one output $\left(y_{1}\right)$ should be regulated to a set point while the other outputs $\left(y_{2} \& y_{3}\right)$ are constrained within certain limits. This fact makes the granulation process an excellent candidate for reference updating method. Figure 3 depicts the fundamental concept of the reference updating method. Basically, simultaneous change of the reference of the unregulated output as a function of $\mathrm{u}$, can limit the control action within certain bounds, such that

$$
r=K_{r} f(u)
$$

The design of the proportional gain $K_{r}$ is dictated by the steady state input-output relationship in Eq. (6). Basically, $K_{r}$ is designed in such a way that when the control action $u$ is approaching towards one of the bounds, the reference is adjusted accordingly to drive the control action in the opposite direction.

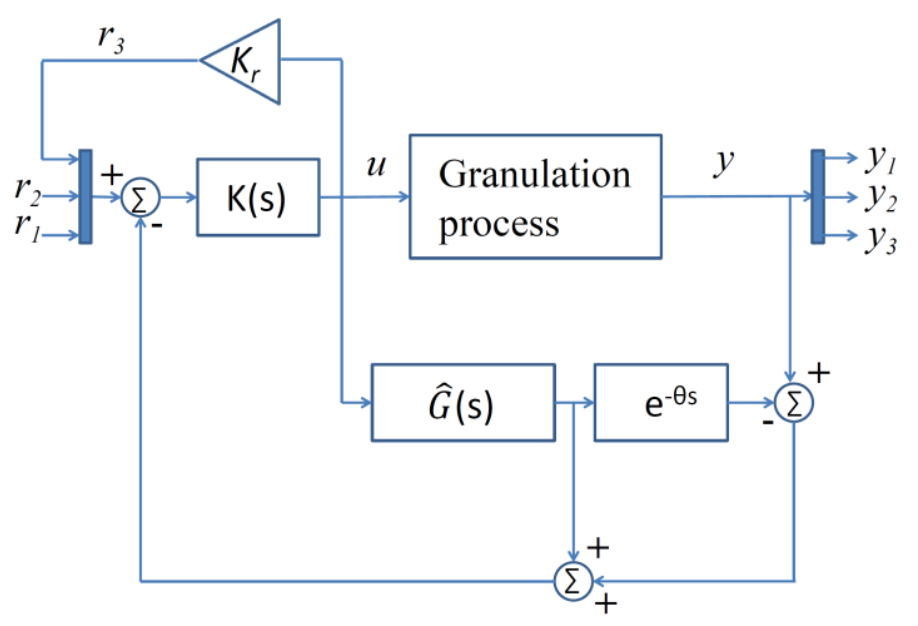

Fig. 3 Closed-loop control structure with Smith predictor and reference updating.

$\left[\begin{array}{l}y_{1} \\ y_{2} \\ y_{3}\end{array}\right]_{S S}=\left[\begin{array}{lll}0.20 & 0.58 & 0.35 \\ 0.25 & 1.10 & 1.30 \\ 0.30 & 0.70 & 1.20\end{array}\right]\left[\begin{array}{l}u_{1} \\ u_{2} \\ u_{3}\end{array}\right]_{S S}$

Assuming zero steady state error $\left(\mathrm{r}=\mathrm{y}_{\mathrm{ss}}\right)$.

$$
\left[\begin{array}{l}
u_{1} \\
u_{2} \\
u_{3}
\end{array}\right]_{s s}=\left[\begin{array}{ccc}
5.1282 & -5.6410 & 4.6154 \\
1.1257 & 1.6886 & -2.1576 \\
-1.9387 & 0.4253 & 0.9381
\end{array}\right]\left[\begin{array}{l}
r_{1} \\
r_{2} \\
r_{3}
\end{array}\right]
$$

For example, if the goal is to minimize $u_{1}$ in the case of positive $r_{1}$, one possibility is the following: $r_{2}$ and $r_{3}$ should be chosen to be positive and negative, respectively, as shown from the first raw of Eqn. (7).

\section{Results and Discussion}

Assessment of the performance of the proposed control approach requires classic closed-loop simulations of the granulation process. The MIMO PI controller parameters were designed as was discussed here. The PI controller parameters are as follows: 


$$
K(s)=\left[\begin{array}{ccc}
\frac{2(2 s+1)}{2 s} & 0 & 0 \\
0 & \frac{2.27(3 s+1)}{3 s} & 0 \\
0 & 0 & \frac{2.083(4 s+1)}{4 s}
\end{array}\right]
$$

The closed-loop simulations of the wet granulation process with the Smith predictor-based PI controller and the PI controller are shown in Figure 4. The figure depicts the closed-loop response of the controlled variables $\left(y_{1}, y_{2}\right.$ and $\left.y_{3}\right)$ and the control effort $\left(u_{1}, u_{2}\right.$ and $u_{3}$ ) against step reference for the bulk density $\left(y_{1}\right)$. As shown in Figure 4, the response from the PI controller without prediction is on the verge of instability, which proves the need for prediction in the granulation process. Although the Smith predictor-based PI controller produces an improved performance stable closed-loop response with zero steady-state error, it significantly violates the quality constraint on the first nozzle flow rate $u_{l}$.

Apart from the stability improvements due to prediction, the control constraints problem needs further improvements. Since the control objective is to regulate the bulk density $\left(y_{1}\right)$ while keeping the other controlled variables $y_{2}$ and $y_{3}$ bounded. This gives rise to the reference updating method, which is a good candidate to limit the controller effort in the control of MIMO process as discussed in section 3.2. Assume that $r_{2}=0.6 r_{1}$ and $r_{3}=K_{r} u_{1}$, where $K_{r}=-0.1$, so that $u_{1}$ have negative action on $r_{3}$ and consequently $u_{1}$ is decreased. Figure 5 depicts the closed-loop response of the granulation process with the Smith predictor-based PI controller and reference updating. Obviously, it can be seen from the figure that this approach is successful to limit the control action $u_{1}$ within its bounds. The figure also compares the result from the Smith predictor-based PI controller with closed-loop results for the granulation system using prioritized objective MPC control (Gatzke \& Doyle III, 2001). It is evident that the results from the current method are more superior in many aspects: stabilizations, regulation, respecting quality constraints, etc.

The proposed control approach is examined against reference tracking problem as seen in Figure 6. The controlled variables $\left(y_{1}, y_{2}\right.$ and $y_{3}$ ) were able to track references efficiently, even though the reference $r_{3}$ is a function of the control effort $u_{1}$. Meanwhile, all the control efforts $\left(u_{1}, u_{2}\right.$ and $\left.u_{3}\right)$ were maintained within the quality constraints.

In order to test the controller robustness and the ability of the predictor to cope with the mismatched time-delay. A mismatched model is introduced via perturbing the time-delays $\left(\theta_{\mathbf{j}}\right)$ by $33 \%$ of its nominal values, and the process parameters $\left(k_{i j}, \tau_{i j}\right)$ are increased by $10 \%$ of its nominal values. Figure 6 also compares the closed-loop response based on the nominal model (see Eq. (2)) with closedloop response based on aforementioned mismatch model using the same control technique. In one hand, the figure shows that the proposed controller is robust against the specified model mismatch. But, on the other hand the control effort $u_{1}$ is trying to violate its lower bound due to model uncertainty. Hence, the robustness of such approach still needs further investigations.
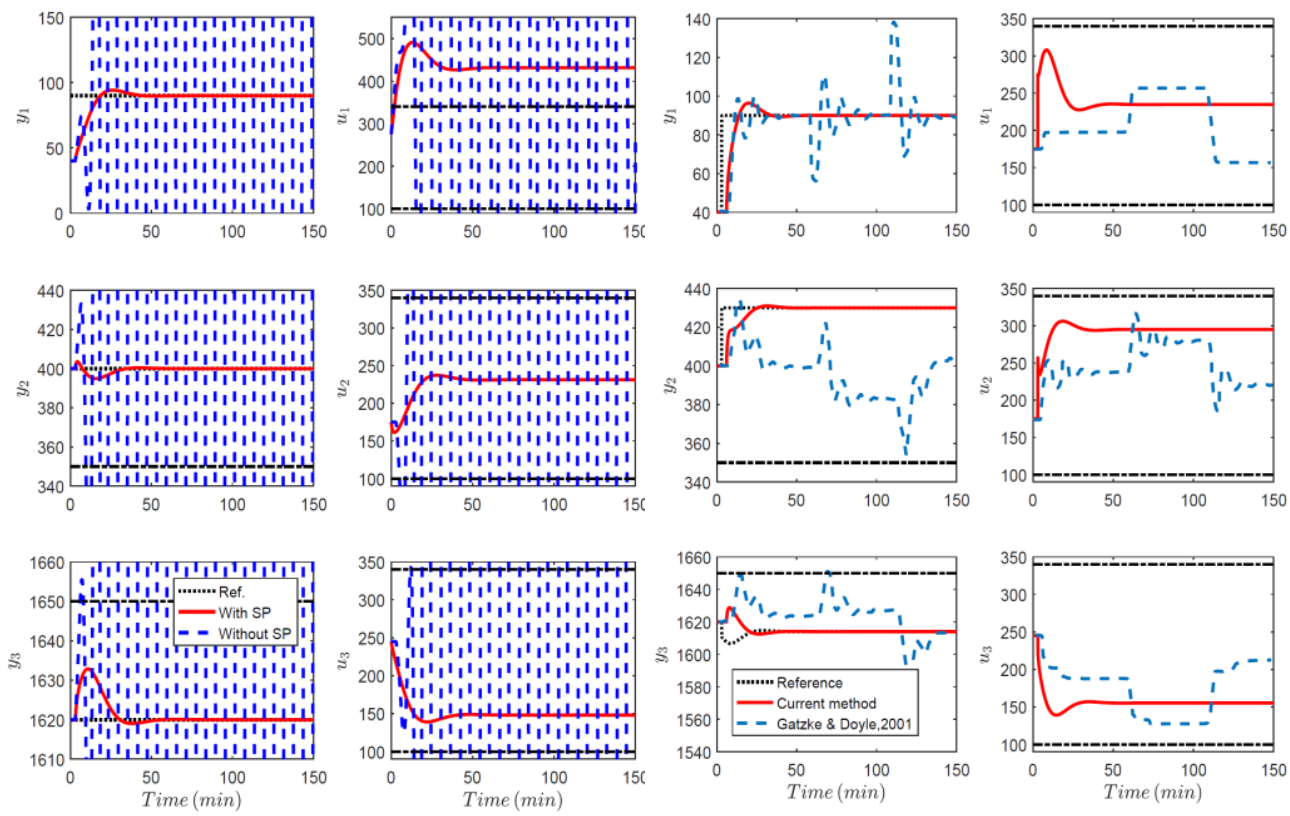

Fig. 4 Closed-loop response of the granulation system with/without Smith predictor.
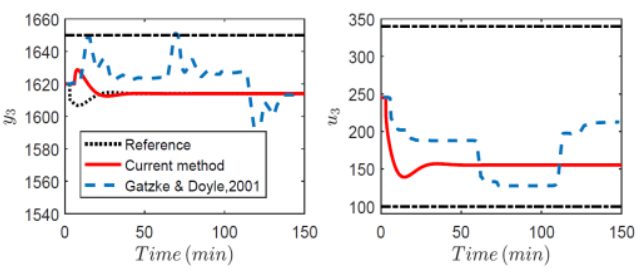

Fig. 5 Closed-loop response of the granulation system with SP and reference updating compared to MPC (Gatzke \& Doyle, 2001). 

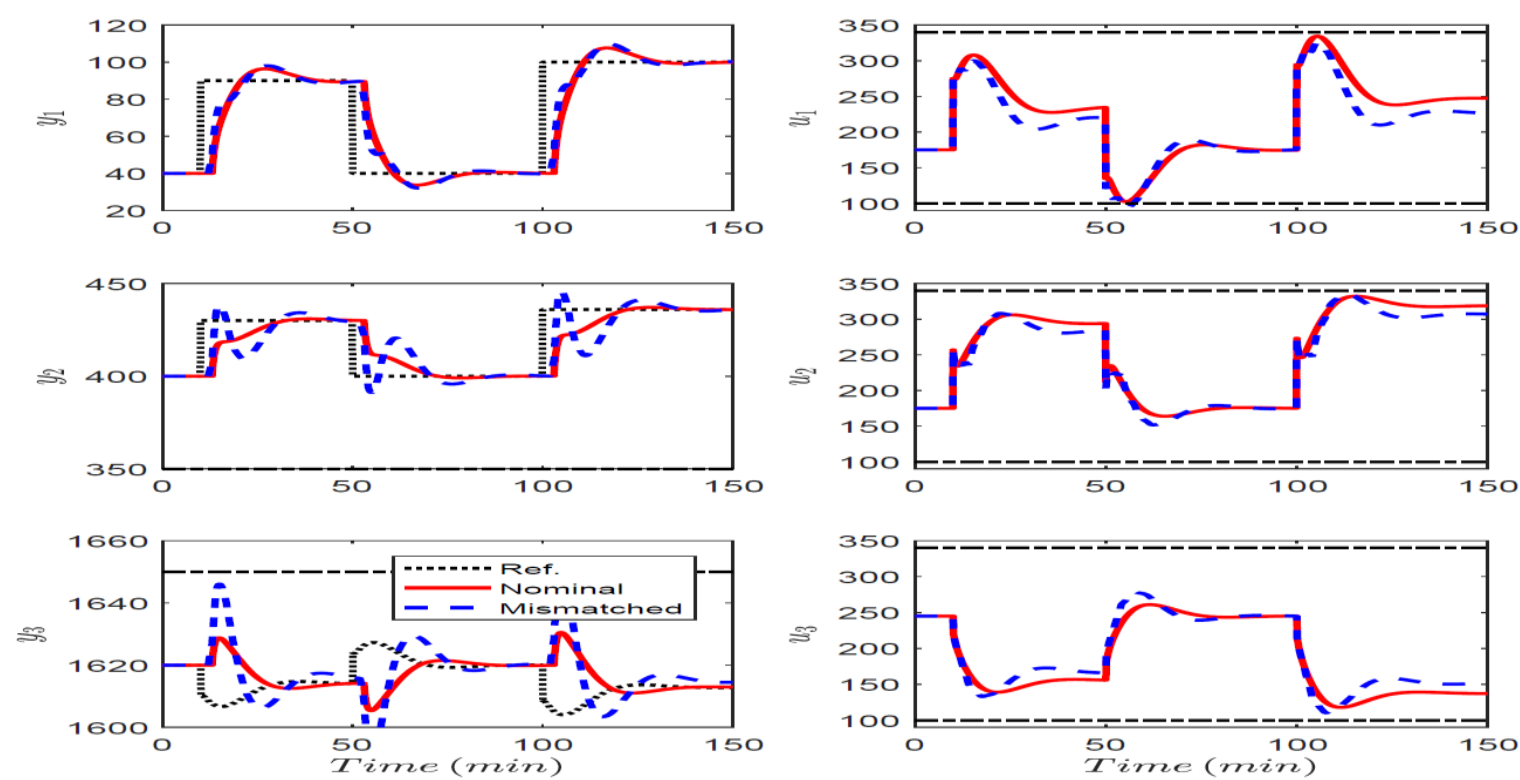

Fig. 6 Aspect ratio closed-loop response of the granulation system with SP and reference updating for the nominal and mismatched case.

\section{Conclusions}

A successful MIMO controller design of a wet granulation process is achieved. The MIMO control approach involves the design of Smith predictor-based PI controller for dead-time compensation. A long with the reference updating method that is introduced to cope with the input/output physically imposed constraints. The performance of the suggested control approach is examined and verified through closed-loop simulations. The proposed control approach was found more efficient than other widely used control techniques for the granulation process such as model predictive control. Although the promising model uncertainty robustness of the controller is achieved, a complete robustness analysis still needs further investigations in the future work.

\section{Nomenclature}

\begin{tabular}{|c|c|c|}
\hline$G$ & $=$ controller gain & {$[-]$} \\
\hline$G(s)$ & $=$ granulation model transfer function & {$[-]$} \\
\hline$\widehat{G}(s)$ & $=$ rational part of $\mathrm{G}(\mathrm{s})$ & {$[-]$} \\
\hline$k$ & $=$ steady state gain & {$[-]$} \\
\hline$K$ & $=$ controller transfer function & {$[-]$} \\
\hline$K_{r}$ & $=$ reference updating gain & {$[-]$} \\
\hline$r$ & $=$ reference & {$[-]$} \\
\hline$s$ & $=$ Laplace operator & {$[-]$} \\
\hline$T$ & $=$ integral time & [min] \\
\hline $\mathrm{u}_{1,2,3}$ & $=$ dimensionless nozzles flow rate & {$[-]$} \\
\hline$y$ & $=$ dimensionless model output & {$[-]$} \\
\hline$\hat{y}$ & $=$ dimensionless predicted output & {$[-]$} \\
\hline$y_{1}$ & $=$ dimensionless bulk density of the product slurry & {$[-]$} \\
\hline$y_{2}$ & $=$ product particle size in the $5^{\text {th }}$ percentile & {$[-]$} \\
\hline$y_{3}$ & $=$ product particle size in the $90^{\text {th }}$ percentile & {$[-]$} \\
\hline$y_{\text {ss }}$ & $=$ steady state output & {$[-]$} \\
\hline$\tau$ & $=$ time constant & [min] \\
\hline$\theta$ & $=$ dead time & [min] \\
\hline$\hat{\theta}$ & $=$ nominal dead time & [min] \\
\hline
\end{tabular}

\section{References}

Buck, A., Palis, S., and E. Tsotsas " Model-based control of particle properties in fluidized bed spray granulation", Powder Technology, 270, 575-583 (2015) Burggraeve, A., Monteyne, T., Vervaet, C., Remon, J. and T. De Beer "Process analytical tools for monitoring, understanding, and con-trol of pharmaceutical fliuidized bed granulation: a review", European Journal of Pharmaceutics and Biopharmaceutics, 83, 2-15 (2013) 
Burggraeve, A., Silva, A., Van Den Kerkhof, T., Hellings, M., Vervaet, C., Remon, J., Vander Heyden, Y., and T. De Beer "Development of a uid bed granulation process control strategy based on real-time process and product measurements". Talanta,100, 293-302 (2012)

Cameron, I. T., Wang, F. Y., Immanuel, C. D., and F. Stepanek "Process systems modelling and applications in granulation: A review", Chem. Eng. Sci., 60, $3723-3750(2005)$

Gatzke, E. P. , and F. J. Doyle III "Model predictive control of a granulation system using soft output constraints and prioritized control objectives", Powder Tech., 121, 149-158 (2001)

Goodwin G. C., Seron M. M., and J. A. De Don "Constrained Control and Estimation: An Optimization Approach", Springer, London, (2005).

Hansuld, E. M. , and L. Briens "A review of monitoring methods for pharmaceutical wet granulation", Inter. Jour. of pharmaceutics, 472, 192-201 (2014)

Iveson, S. M., Litster, J. D., Hapgood, K. , and Ennis, B. J., "Nucleation, growth and breakage phenomena in agitated wet granulation processes: A review", Powder Tech., 117, 3-39 (2001)

Landau I. D., Lozano R., M’Saad M., and A. Karim "Adaptive Control: Algorithms, Analysis and Applications", Springer, New York, (2011)

Merkus, H. G., and Meesters, G. M., "Production, Handling and Characterization of Particulate Materials", Springer, (2016)

Normey-Rico, J. E.;"Control of Dead-Time Processes", Springer Science , Business Media, (2007)

Petrovic, J., Chansanroj, K., Meier, B., Ibric, S. and G. Betz "Analysis of fluidized bed granulation process using conventional and novel modeling techniques", Euro. Jour. of Pharmaceutical Sci., 44, 227-234 (2011)

Pottmann, M., Ogunnaike, B. A., Adetayo, A. A., and B. J. Ennis "Model-based control of a granulation system", Powder Tech., 108, 192-201 (2000)

Rankell, A. S., Scott, M. W., Lieberman, H. A., Chow, F. S., and J. V. Battista, "Continuous production of tablet granulations in a fluidized bed II. operation and performance of equipment", Jour. of Pharmaceutical Sci., 53, 320-324 (1964)

Reynolds, G. K., Fu, J. S., Cheong, Y. S., Hounslow, M. J., and A. D. Salman "Breakage in granulation: a review", Chem. Eng. Sci., 60, 3969-3992 (2005)

Sanders, C. F., Hounslow, M. J., and F. J. Doyle III "Identification of models for control of wet granulation", Powder Tech., 188, 255-263 (2009)

Singh, R., Barrasso, D., Chaudhury, A., Sen, M., Ierapetri-tou, M., and R. Ramachandran "Closed-loop feedback control of a continuous pharmaceutical tablet manufacturing process via wet granulation", Jour. of Pharmaceutical Innov., 9, 16-37 (2014)

Smith, O. J. M., "Posicast control of damped oscillatory systems", Proceedings of the IRE, 45, 1249-1255 (1957)

Suresh, P., Sreedhar, I., Vaidhiswaran, R., and A. Venugopal "A comprehensive review on process and engineering aspects of pharmaceutical wet granulation", Chem. Eng. Jour., 328, 785-815 (2017)

Tardos, G. I., Khan, M. I., and P. R. Mort "Critical parameters and limiting conditions in binder granulation of ne powders", Powder Tech., 94, 245-258 (1997) 